\title{
Development of auditory, language, and speech skills of children with cochlear implants: a study of aural rehabilitation benefits
}

\begin{abstract}
Objectives: to determine the benefits of aural rehabilitation in the auditory, language, and speech development of children with cochlear implants.

Method: This longitudinal study was conducted on 15 deaf children (m.a. 45.27mo) who received cochlear implants in the AmirAlam cochlear implant center in Tehran, Iran. Data on the subjects were collected before they received cochlear implants, and then again 2, 4, 6, and 8months after implantation. The children's developmental age in terms of auditory, speech, and receptive and expressive language skills was determined through interviews with their parents and scored using the Newsha Developmental Scale. We then computed the Pretest Developmental Rate, the Intervention Efficiency Index, and the Proportional Change Index.

Results: A significant difference was observed among the preintervention and the 4 follow-up assessments of the Pretest Developmental Rate in four studied skills $(\mathrm{P}<0.0001)$. There was a significant difference among the 4 follow-up assessments of the Intervention Efficiency Index and also the Proportional Change Index in all studied skills ( $\mathrm{P} \leq 0.043, \mathrm{P} \leq 0.001$; respectively).

Conclusion: After cochlear implants, the long-term aural rehabilitation for accelerating the auditory, speech, and language development is required until deaf children achieve age-appropriate skills.
\end{abstract}

Keywords: cochlear implants, intervention, auditory skills, spoken language, speech
Volume II Issue 4 - 2019

\author{
Zahra Jeddi,' Aziz Kassani ${ }^{2}$ \\ 'Department of Audiology, Shiraz University of Medical Sciences, \\ Iran \\ ${ }^{2}$ Department of Epidemiology, Shiraz University of Medical \\ Sciences, Iran
}

Correspondence: Zahra Jeddi, Department of Audiology, School of Rehabilitation Sciences, Shiraz University of Medical Sciences, Iran, Tel +98 7I 3626I08I, Fax +98 7I 36272495, Email z.jeddi.au@gmail.com

Received: June II, 2019 | Published: July 16, 2019

\section{Introduction}

The lack of early auditory input hinders the normal development of the auditory system and the progress of language and speech skills. ${ }^{1}$ Sound pattern learning depends on the neural commitment to the acoustic characteristics of language. Children with congenital sensorineural hearing loss do not experience similar neural commitments, and therefore the normal development of spoken language undergoes various changes. ${ }^{2}$ Given that the speech sounds that constitute languages are perceived through the auditory senses, hearing-impaired children not only exhibit a reduced ability to listen to others but also have difficulty monitoring their own speech production. ${ }^{3}$ They cannot achieve normal acoustic feedback provided by air and bone conduction. Their inability to perceive intensity, frequency and duration, which are characteristics of speech, and their poor segmental performance negatively affect their speech intelligibility. ${ }^{4}$ If the duration of the auditory deprivation is extended, the resultant deeper sensory deprivation leads to insufficient stimulation of the brain, thereby hindering neural development and auditory learning. ${ }^{3}$ The restoration of auditory input allows hearingimpaired children to develop the skills needed for sound recognition and improve their communication skills. ${ }^{5}$ Many hearing-impaired children receive significant benefits from cochlear implants and advanced speech-processing strategies. ${ }^{6}$ Unfortunately, cochlear implant outcomes vary widely, and some children show little improvement in auditory and spoken language skills even after using the device for many years. Research has suggested that there is substantial neuroplasticity in children with cochlear implants. Auditory training is needed to optimize the use of auditory plasticity and the learning of speech patterns. Auditory training is a critical addition to cochlear implantation and enables children to access subtle spectral-temporal cues. ${ }^{7}$

Studies suggest that cochlear implantation and aural rehabilitation enable hearingimpaired children to communicate vocally with others. These vocal responses encourage adults to continue the natural pattern of communication, which further develops children's vocal and auditory communication skills. ${ }^{8}$ The Wu et al., ${ }^{9}$ study (2003) of auditory development in children with cochlear implants documented sustained improvements over two years in auditory skill levels in hearing-impaired children after implantation. ${ }^{9}$ Studies related to the rate of language growth have suggested that some children with cochlear implants acquire spoken language at a normal or near normal rate, which leads to a decrease in the difference between chronological and developmental ages, or at least to a difference that remains constant. In addition, some of the children in these studies learned language at a faster rate than normal and achieved age-appropriate language levels five years after the implantation. ${ }^{10}$ Flipsen (2006), when describing the speech intelligibility of children with cochlear implants, suggested that during the first two years after implantation, intelligible speech begins to appear rapidly and the pattern of development is fairly similar to normal patterns. ${ }^{11}$ 
Recent studies emphasize the importance of cochlear implantation for hearing-impaired children in facilitating the development of auditory, language, and speech skills. The variability in the results of these studies demonstrates the influence of aural rehabilitation on the developmental progress of children with cochlear implants. What is important is the assessment of the developmental skills level of hearing-impaired children with cochlear implants to inform their parents and provide them with realistic expectations. In addition, expert opinion on the expected performance levels of children with hearing aids or cochlear implants may help determine the cochlear implant candidacy of hearing-impaired children. Despite significant attempts to improve speech processing strategies in children with cochlear implants, the importance of aural rehabilitation for these children and its benefits on cochlear implantation outcomes has not been considered in depth. The present study aims to determine the benefits of aural rehabilitation programs in auditory, language, and speech development of children with cochlear implants.

\section{Material and methods}

\section{Participants}

Fifteen hearing-impaired children, including 7 males (46.7percent) and 8 females (53.3percent) who received cochlear implants at the AmirAlam cochlear implant center in Tehran (Iran), participated in this study. At the start of the study, the mean age of the children was $45.27( \pm 14.45)$ months. The data relating to the ages of the children at the time of their hearing loss diagnosis and amplification are shown in Table 1. The mean age of children in this study is high due to restrictions on the importation of cochlear implants into this country and the prolonged waiting time for candidates for cochlear implants. All participants had severe to profound bilateral congenital sensorineural hearing loss, but had no additional disabilities (e.g., developmental delays, motor deficits, attention deficits, learning disabilities, or mental retardation) other than the hearing loss. Auditory neuropathy/ spectrum disorder was ruled out in these children based on diagnostic criteria. They were fitted with bilateral hearing aids with optimal adjustment and provided auditory-oral rehabilitation at least 6months before implantation. The children had normal-hearing parents who used oral communication. We used sequential sampling to sample the children. The study samples were selected based on information related to the audio logical and medical assessments in the Amir Alam cochlear implant center database and through the parents' completion of a medical history form, which included demographic information as well as birth, medical, and hearing loss histories. This study was approved by the ethics committee of the Tehran University of Medical Sciences.

Table I Characteristics of the study participants $(n=15)$

\begin{tabular}{|c|c|c|c|}
\hline Range & $\begin{array}{l}\text { Standard } \\
\text { deviation }\end{array}$ & Mean & The ages of children(month) \\
\hline 17 & 5.70 & 8.53 & Hearing loss diagnosis \\
\hline 22 & 7.64 & 12.20 & Amplification \\
\hline 21 & 7.53 & 13.07 & $\begin{array}{l}\text { Beginning the rehabilitation } \\
\text { program }\end{array}$ \\
\hline 38 & 14.45 & 44.27 & Cochlear implant operation \\
\hline
\end{tabular}

\section{Procedure}

In this longitudinal study, we assessed speech, auditory, and receptive and expressive language skills of hearing-impaired children using the Newsha Developmental Scale. The children's parents were asked to answer questions related to each of the above-mentioned skills using this scale. The assessment was performed before the children received cochlear implants, and then again 2,4,6 and 8months after implantation. The aural rehabilitation program used auditoryverbal communication to train the hearing-impaired children with cochlear implants. The children underwent auditory training by an audiologist skilled in cochlear implant rehabilitation for 2 one-hour sessions per week. They were also trained in language and speech skills by an expert speech therapist for 2 one-hour sessions per week. In each session, while performing the intervention with the child, the therapist trained the parents to do the practices at home. Given the ethical considerations and the cochlear implant center regulations, we did not implement a control group with an alternative rehabilitation program or no program.

Newsha developmental scale: This is a Persian norm-referenced assessment designed for children 0-6years of age. It assesses the main developmental areas of auditory, speech, receptive and expressive language, cognition, social communication, and motor skills in 13 age ranges including: level 1 (0-3months); level 2 (4-6months); level 3 (7-9 months); level 4 (10-12months); level 5 (13-15months); level 6 (16-18months); level 7 (19-24 months); level 8 (25-30months); level 9 (31-36months); level 10 (37-42months); level 11 (43-48months); level 12 (49-60 months); and level 13 (61-72months). This scale is intended to determine whether the development trajectory of each of the developmental areas is normal or delayed. The child's progress curve during the rehabilitation program can be depicted by the implementation of this scale. Interpretation of the results is based on minimum and maximum scores, with each item scoring one point. If a child achieves the minimum score or above, then the child is said to have age-appropriate developmental skills. However, if the child scores less than the minimum over two assessments separated by less than one week, their developmental delay can be calculated by the completion of the scale for lower age ranges. The reliability of this scale is $95 \%$ and its content validity index for various skills across age groups is between 0.8 and $1 .{ }^{12}$

Calculation: For each time interval, the child's score in each of the studied skills was determined based on the parent's response to items on the Newsha Developmental Scale. For each skill, the developmental age was determined by the upper limit of the age range in which the child achieved the required score. The Pretest Developmental Rate (PDR) was then calculated by dividing the pretest developmental age by the pretest chronological age. This index shows the rate of development in the pre-intervention interval. ${ }^{13}$ For the time intervals following cochlear implantation, the Intervention Efficiency Index (IEI) and the Proportional Change Index (PCI) were computed to determine the efficiency of the aural rehabilitation program. The IEI was calculated by dividing the difference between the pre- and posttest developmental ages by the time interval between the pre-and post-tests. This index revealed the rate of development during the intervention. The PDR was then divided by the IEI, which resulted in the PCI, which is indicative of the efficiency index of the aural rehabilitation program $(\mathrm{PCI}=\mathrm{PDR} / \mathrm{IEI}) .^{13}$

\section{Data analysis}

The normality of the data was assessed with the KolmogorovSmirnov test. We then used the nonparametric Friedman test for the comparison between the preintervention and the four follow-up 
assessments of PDR and also for the comparison between the four follow-up assessments of IEI and PCI in each of the four studied skills. Data analysis was performed using SPSS 18.0 and $\mathrm{P}<0.05$ was considered significant.

\section{Results}

\section{Developmental age}

After the cochlear implantation and during the aural rehabilitation, the mean developmental age of the participants gradually increased and approached their chronological age. Figure 1 show the mean chronological and developmental ages for all time intervals for the assessment of auditory, speech, and receptive and expressive language skills.

\section{Pretest developmental rate}

Figure 2 illustrates the results of the PDR for all time intervals for the assessment of auditory, speech, and receptive and expressive language skills. A significant increase was observed in the PDR during the aural rehabilitation period $(\mathrm{P}<0.0001)$

\section{Intervention efficiency index}

The mean and standard deviation of the IEI in auditory, speech, and receptive and expressive language skills during the four followup assessments is shown in Figure 3. According to the statistical analysis, there was a significant difference among the four followup assessments of the IEI in auditory, speech, and receptive and expressive language skills $(\mathrm{P} \leq 0.043)$.

\section{Proportional change index}

Figure 4 illustrates the results of the Proportional Change Index (PCI) calculation for auditory, speech, receptive, and expressive language skills at the first, second, third, and fourth follow-up assessments. There was a significant difference among the four follow-up PCI assessments in each of the studied skills $(\mathrm{P} \leq 0.001)$.

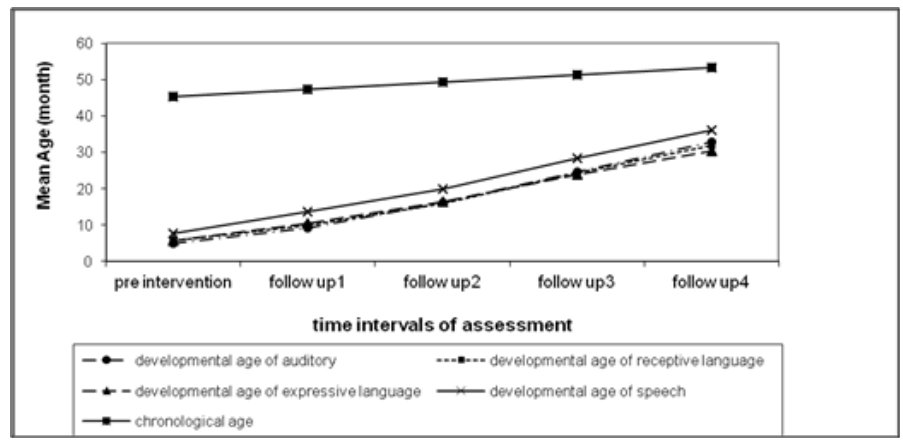

Figure I The chronological and developmental ages of auditory, speech, and receptive and expressive language skills at pre-intervention and at 4 follow-up assessments.

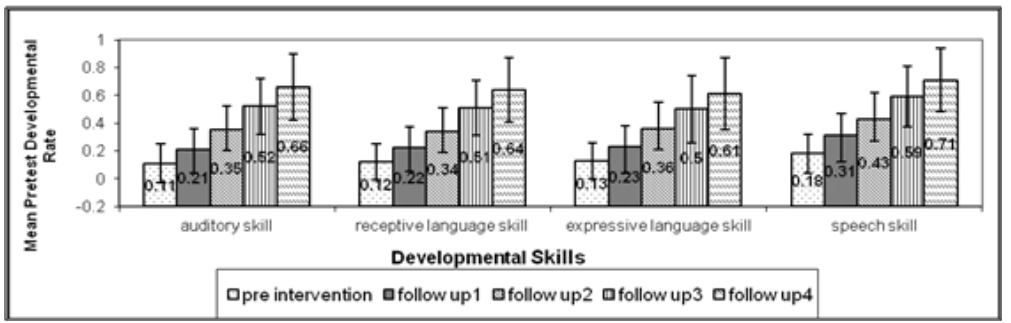

Figure $\mathbf{2}$ The mean and standard deviation of PDR at pre-intervention and at 4 follow-up assessments in auditory, speech, and receptive and expressive language skills.

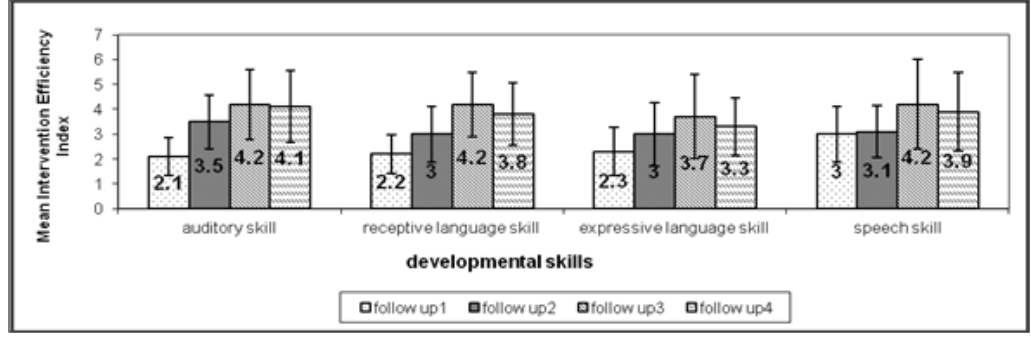

Figure 3 The mean and standard deviation of IEl at 4 follow-up assessments in auditory, speech, and receptive and expressive language skills. 


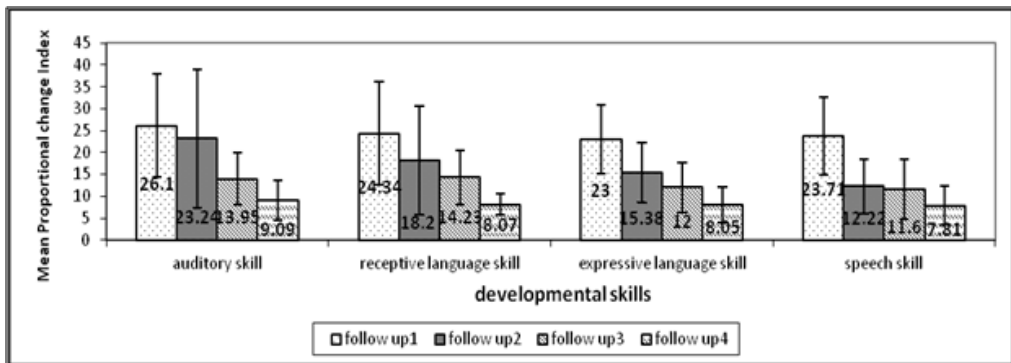

Figure 4 The mean and standard deviation of PCI at 4 follow-up assessments in auditory, speech, and receptive and expressive language skills.

\section{Discussion}

In this study, a remarkable difference was observed between the chronological and developmental age of the hearing-impaired children in auditory, language, and speech skills before cochlear implantation and aural rehabilitation. This suggests that auditory deprivation in early childhood can result in developmental delays in auditory, language, and speech skills. Following cochlear implantation and after 8 months of aural rehabilitation, the developmental age of these children approached their chronological age. Nevertheless, these children did not show age-appropriate developmental skills by the end of the aural rehabilitation program. The finding of improvement in children's performance following implantation and rehabilitation is consistent with previous studies. ${ }^{14,15}$ Similar to this result, Schramm et al., ${ }^{16}$ reported that children with cochlear implants showed considerable progress in auditory behaviors and expressive vocabulary between the chronological ages of 24 and 36months and approach the skills of normal-hearing children within 6 months of receiving implants. ${ }^{16}$ Nicholas et al. ${ }^{17}$ in a study of the vocabulary of 76 children with cochlear implants, showed that children who received cochlear implants by $12-13$ months of age achieved age-appropriate vocabulary by 4.5 years of age. ${ }^{17}$ A study by Bakhshaee et al. ${ }^{4}$ of 47 children with cochlear implants measured speech intelligibility at yearly intervals for 5 years after the implantation and reported that there was considerable improvement in the speech intelligibility of these children because of the use of this device. ${ }^{4}$ Cochlear implants allow hearing-impaired children to access sound and aural rehabilitation facilitates their use of this auditory input to improve their developmental skills. As children use the cochlear implants and aural rehabilitation, their developmental skills improve, resulting in the approximation of the developmental age to the chronological age. The presence of a gap between the developmental and chronological ages after 8months of aural rehabilitation in the present study calls for longer-term aural rehabilitation programs in children with cochlear implants until they are able to achieve age-appropriate skills.

The PDR provides valuable information about the child's progress in each developmental skill and represents the delayed development in hearing-impaired children. In the present study, the mean PDR was less than 1 before the cochlear implantation. After cochlear implantation and implementation of aural rehabilitation, the mean PDR increased and gradually approached 1 . This is in accordance with the results of other studies such as the study by May-Mederake et al. ${ }^{18}$ that observed accelerated progress in auditory skills for the first 9 months after the device was implanted. ${ }^{18}$ Svirsky et al., ${ }^{19}$ conducted a 30 -months study on the language abilities of 70 children with cochlear implants. The authors concluded that the mean rate of spoken language development in children with cochlear implants is close to that of children with normal hearing. ${ }^{19}$

In the present study, the rate of the children's development during the intervention period was examined by the IEI. An IEI greater than 1 at each follow-up assessment suggested that, after using the cochlear implant and aural rehabilitation, developmental skills in the hearing-impaired children progressed at a greater rate than that of children with normal hearing. Similarly, a study by Schramm et al., ${ }^{16}$ reported faster progress of children with cochlear implants in the first months of hearing compared with children with normal hearing. ${ }^{16}$ On the other hand, a study by Nott et al., ${ }^{20}$ on the acquisition of first words and first word combinations in two groups of children, one with normal hearing and the other with cochlear implants, stated that the development curve slope for children with normal hearing is steeper than that of children with cochlear implants. ${ }^{20}$ Since some participants in the Nott study underwent reimplantation, the interrupted acquisition process probably resulted in the conflict between the Nott study and the other studies. The increased IEI in this study is indicative of the effectiveness of aural rehabilitation on the development of auditory, language, and speech skills of children with cochlear implants. Thus, the developmental skills of children with cochlear implants progress at a faster rate than that expected of normal-hearing children, which enables children with cochlear implants to achieve age-appropriate skills. Although the IEI for the last interval of the assessment showed lower values than the IEI of the previous interval, the mean IEI was still greater than 1 . Given that the children with cochlear implants achieved a level of auditory comprehension after $6-7$ months of aural rehabilitation, the decline in the IEI at the fourth interval may be attributable to the complexity of auditory tasks and the subsequent increase in the level of complexity of language and speech skills, which in turn lead to a decelerated rate of development. As noted by $\mathrm{Lu}$ and Qin, ${ }^{21}$ the post-implant performance in simple auditory tasks like sound detection improved at a faster rate compared to that in complex tasks such as sound recognition. ${ }^{21}$

The PCI determines the efficiency of aural rehabilitation programs, and in the present study, the mean PCI for each of the studied skills at all assessment time intervals was greater than 1. This suggests that aural rehabilitation is effective for children with cochlear implants because the rate of development during the intervention was greater than the rate of development during the pre-intervention. In accordance with this finding, the study by Li et al.,22 on the outcomes of facilitated aural rehabilitation for children with cochlear implants mentioned an improved rate of auditory comprehension development after implantation. ${ }^{22}$ Geers et al., ${ }^{23}$ suggested that the implementation of aural rehabilitation after cochlear implantation facilitates access 
by hearing-impaired children to age-appropriate spoken language levels. ${ }^{23}$ In the present study, a remarkable decline in the PCI was seen during the aural rehabilitation program. In contrast to this finding, a study by Jing et al. ${ }^{24}$ on the validation of rehabilitation programs in children with cochlear implants revealed that the effectiveness of an aural rehabilitation program increased with the length of the program. ${ }^{24}$ Given the poor developmental skills of our study's children before they received cochlear implants, the accelerated development of skills in the first 2 months of aural rehabilitation after cochlear implantation may cause the impact of the rehabilitation program on the development of skills to appear remarkable. Therefore, despite the high rate of development in these children compared to children with normal hearing, the relative change observed in the developmental skills became smaller than that in the previous stage during the intervention. $\mathrm{Lu}$ and $\mathrm{Qin}^{21}$ have also noted the more rapid development rate of auditory abilities in the first versus second year of implantation. ${ }^{21}$

The present study was conducted on 15 hearing-impaired children who received cochlear implants at the Amir Alam cochlear implant center. The limitations of this study include the small sample size and short period of follow-up. Given that the families of hearingimpaired children are referred to the cochlear implant center from various regions of Iran, and due to poor cooperation from the other families, it is difficult to follow-up larger groups of children for a longer period of time. Due to the interference of parent perspectives in the developmental skills progress of their child, the study of the progress of developmental skills of children through parents' completion of the Newsha Developmental scale results in a bias on the part of the parents. However, we attempted to ensure that the parents understood each item of the scale correctly through sufficient and detailed illustration of those items. Continued research at regular time intervals may provide more information on the benefits of aural rehabilitation and the successful results of cochlear implant use.

\section{Conclusion}

The present study examined the development rate of auditory, speech, and receptive and expressive language skills of Persian hearing-impaired children following cochlear implants as measured by the Newsha Developmental Scale, for the purpose of determining the benefits of an aural rehabilitation program. The results of this study revealed the necessity for long-term aural rehabilitation for hearing-impaired children with cochlear implants to accelerate their development of auditory, language, and speech skills. This study provides a criterion for evaluating the benefits of interventions for cochlear implant experts so that they can design the optimal aural rehabilitation programs for children with cochlear implants. In addition, the depiction of the child's progress curve based on this study's method will help counsel parents and provide them with realistic expectations. We hope that documentation of the development trajectory of auditory, language, and speech skills of Persian children with cochlear implants compared with previous studies will help reduce the age of cochlear implantation.

\section{Funding details}

None.

\section{Acknowledgments}

None.

\section{Conflict of interest}

We declare there is no financial interest or any conflict of interest.

\section{References}

1. Kim L, Jeong S, Lee Y, et al. Cochlear implantation in children. Auris nasus larynx. 2009;37(1):6-17.

2. Niparko J, Tobey E, Thal D, et al. Spoken Language Development in Children Following Cochlear Implantation. JAMA. 2010;303(15):1498.

3. Sevinc S, Ozcebe E, Atas A, et al. Articulation skills in Turkish speaking children with cochlear implant. Int $J$ Pediatr Otorhinolaryngol. 2009;73(10):1430-1433.

4. Bakhshaee M, Ghasemi M, Shakeri M, et al. Speech development in children after cochlear implantation. Eur Arch Otorhinolaryngol. 2007;264(11):1263-1266.

5. Lin F, Wang N, Fink N, et al. Assessing the use of speech and language measures in relation to parental perceptions of development after early cochlear implantation. Otol Neurotol. 2008;29(2):208.

6. Levine D, Strother Garcia K, Golinkoff RM, et al. Language development in the first year of life: What deaf children might be missing before cochlear implantation. Otol Neurotol. 2016;37(2):e56-e62.

7. Fu Q, Galvin III J. Maximizing cochlear implant patients' performance with advanced speech training procedures. Hear Res. 2008;242(12): $198-208$

8. Tait M, De Raeve L, Nikolopoulos TP. Deaf children with cochlear implants before the age of 1 year: Comparison of preverbal communication with normally hearing children. Int $J$ Pediatr Otorhinolaryngol. 2007;71(10):1605-1611.

9. Wu JL, Yang HM. Speech perception of Mandarin Chinese speaking young children after cochlear implant use: effect of age at implantation. Int J Pediatr Otorhinolaryngol. 2003;67(3):247-53.

10. Duchesne L, Sutton A, Bergeron F. Language Achievement in Children Who Received Cochlear Implants Between 1 and 2 Years of Age: Group Trends and Individual Patterns. J Deaf Stud Deaf Educ. 2009;14(4):465-485.

11. Flipsen P. Intelligibility of conversational speech produced by children with cochlear implants. J Commun Disord. 2006;39(2):93-108.

12. Jafari Z, Malayeri S. The psychometric properties of Newsha Developmental Scale: an integrated test for Persian speaking children. Iran J Pediatr. 2012;22(1):53-59.

13. McNamara R, Johnson D, Barringer D. The SKI*HI Manual. Logan: HI Institute; 1994. p. 19-21.

14. Percy Smith L, Tonning TL, Josvassen JL, et al. Auditory verbal habilitation is associated with improved outcome for children with cochlear implant. Cochlear Implants Int. 2018;19(1):38-45.

15. Goh BS, Fadzilah N, Abdullah A, et al. Long-term outcomes of Universiti Kebangsaan Malaysia Cochlear Implant Program among pediatric implantees. Int J Pediatr Otorhinolaryngol. 2018;105:27-32.

16. Schramm B, Bohnert A, Keilmann A. Auditory, speech and language development in young children with cochlear implants compared with children with normal hearing. Int $J$ Pediatr Otorhinolaryngol. 2010;74(7):812-819.

17. Nicholas J, Geers A. Expected test scores for preschoolers with a cochlear implant who use spoken language. Am J Speech Lang Pathol. 2008;17(2):121.

18. May Mederake B, Kuehn H, Vogel A, et al. Evaluation of auditory development in infants and toddlers who received cochlear implants 
under the age of 24 months with the LittlEARS $®$ Auditory Questionnaire. Int J Pediatr Otorhinolaryngol. 2010;74(10):1149-1155.

19. Svirsky M, Robbins A, Kirk K, et al. Language development in profoundly deaf children with cochlear implants. Psych Sci. 2000;11(2):153.

20. Nott P, Cowan R, Brown PM, et al. Early language development in children with profound hearing loss fitted with a device at a young age: part I-the time period taken to acquire first words and first word combinations. Ear Hear. 2009;30(5):526-540.

21. Lu X, Qin Z. Auditory and language development in Mandarin-speaking children after cochlear implantation. Int $J$ Pediatr Otorhinolaryngol. 2018;107:183-189.
22. Li H, Guo Y, Zheng X, et al. The outcomes of the facilitated aural rehabilitation for children with cochlear implantation. Chinese Scientific Journal of Hearing and Speech Rehabilitation. 2008;4:11.

23. Geers AE. Factors influencing spoken language outcomes in children following early cochlear implantation. Adv Otorhinolaryngol. 2006;64:50-65.

24. Jing Liuqzhz, Yuxi Wxliyguo, Fang Y. Validation of rehabilitation and relative analysis for prelingually deafened children with cochlear implant. J Clinic Otorhinolaryngol Head Neck Surg. 2011;25(13):582-584. 